\title{
$a$-Points of the Riemann zeta-function on the critical line
}

\section{Stephen J. Lester}

Department of Mathematics, University of Rochester, 915 Hylan Building, RC Box 270138, Rochester, NY 14627, USA

Correspondence to be sent to: lester@math.rochester.edu

We investigate the proportion of the nontrivial roots of the equation $\zeta(s)=a$, which lie on the line $\mathfrak{R} s=1 / 2$ for $a \in \mathbb{C}$ not equal to zero. We show that at most one-half of these points lie on the line $\mathfrak{R} s=1 / 2$. Moreover, assuming a spacing condition on the ordinates of zeros of the Riemann zeta-function, we prove that zero percent of the nontrivial solutions to $\zeta(s)=a$ lie on the line $\mathfrak{R} s=1 / 2$ for any nonzero complex number $a$.

\section{Introduction}

Let $s=\sigma+i t$ be a complex variable, $\zeta(s)$ be the Riemann zeta-function, and $a$ be a nonzero complex number. The solutions to $\zeta(s)=a$, which we will denote by $\rho_{a}=\beta_{a}+i \gamma_{a}$, are called $a$-points, and their distribution has been widely studied. For principal references see [16], [9], and [13].

For every $a$ there is a $n_{0}(a)$ so that for all $n \geq n_{0}$ there is an $a$-point of $\zeta(s)$ quite close to $s=-2 n$. Moreover, in the half-plane $\sigma \leq 0$ there are only finitely many other $a$-points; and we call the $a$-points with real part $\leq 0$ trivial $a$-points. The remaining $a$-points all lie in a strip $0<\sigma<A$, where $A$ depends on $a$, and are called nontrivial $a$-points. Let $\rho_{a}=\beta_{a}+i \gamma_{a}$ denote a nontrivial $a$-point. The number of these is given by

$$
N_{a}(T)=\sum_{1<\gamma_{a} \leq T} 1=\frac{T}{2 \pi} \log \frac{T}{2 \pi}-\frac{T}{2 \pi}+O(\log T),
$$

for $a \neq 1$ (this holds for $a=0$ as well). In the case $a=1$ there is an additional $-(\log 2) T /(2 \pi)$ term on the right-hand side of the equation (see Levinson [9]).

By analogy with the case $a=0$, it is natural to investigate the distribution of the nontrivial $a$-points. Let

$$
N_{a}\left(\sigma_{1}, \sigma_{2} ; T\right)=\sum_{\substack{0<\gamma_{a} \leq T \\ \sigma_{1}<\beta_{a}<\sigma_{2}}} 1
$$

For fixed $1 / 2<\sigma_{1}<\sigma_{2}<1$ and $a \neq 0$ Borchsenius and Jessen [5] showed there exists a constant $C\left(a, \sigma_{1}, \sigma_{2}\right)>0$ such that

$$
N_{a}\left(\sigma_{1}, \sigma_{2} ; T\right)=C\left(a, \sigma_{1}, \sigma_{2}\right) T+o(T) \quad(T \rightarrow \infty) .
$$

As for the case $a=0$, it is well-known that there can be at most $\ll T^{\theta\left(\sigma_{1}\right)}$, with $\theta\left(\sigma_{1}\right)<1$, in such a strip (for instance, see Chapter IX of $[16])$.

Levinson [9] studied $a$-points near the critical line and showed that for any $\delta>0$

$$
\sum_{\substack{0<\gamma_{a} \leq T \\ 1 / 2-\delta<\beta_{a}<1 / 2+\delta}} 1=\frac{T}{2 \pi} \log T+O_{\delta}(T),
$$

where the implied constant depends on $\delta$. From (1.1), it immediately follows that $N_{a}(T)(1+o(1)) a$-points of $\zeta(s)$ lie in the strip $1 / 2-\delta<\sigma<1 / 2+\delta, 1<t<T$, for any fixed $\delta$. Therefore, the $a$-points of $\zeta(s)$ cluster near the critical line $\sigma=1 / 2$. 
Selberg also studied the distribution of the $a$-points of $\zeta(s)$ near the critical line. Under the assumption of the Riemann hypothesis, Selberg, in unpublished work, showed for $c>0$ and $\sigma=1 / 2-c \sqrt{\pi \log \log T} / \log T$ that

$$
\sum_{\substack{1<\gamma_{a} \leq T \\ \beta_{a}>\sigma}} 1=\int_{-c}^{\infty} e^{-\pi x^{2}} d x N_{a}(T)(1+o(1))
$$

(a proof may be found in Tsang's thesis [17]). By this we see that, assuming the Riemann hypothesis, about $1 / 2$ of the $a$-points of $\zeta(s)$ lie to the left of the line $\sigma=1 / 2$ at distances of order $\sqrt{\log \log T} / \log T$. Taking $c \rightarrow 0^{+}$ slowly, it follows that

$$
N_{a}(0,1 / 2 ; T) \geq \frac{1}{2} \cdot N_{a}(T)(1+o(1)) .
$$

Understanding the distribution of the remaining one-half of the $a$-points seems to be quite difficult. Selberg [13] states that most of these points lie quite close to the critical line at distances of order not exceeding $(\log \log \log T)^{3} /(\log T \sqrt{\log \log T})$ away from the critical line. Moreover, he conjectured that approximately one half of these lie to the left of the line $\sigma=1 / 2$ while the other half lie to the right. That is, three-quarters of the nontrivial $a$-points lie to the left of the critical line $\sigma=1 / 2$, while the remaining one-quarter lie to the right of the line.

On the critical line $\sigma=1 / 2$ we expect that there are very few $a$-points. In fact, Selberg [13] has conjectured that there are at most only finitely many $a$-points on the critical line. Observe that for almost all $a$ there are no $a$-points of $\zeta(s)$ with $\beta_{a}=1 / 2$. This is because the set $\left\{\zeta\left(\frac{1}{2}+i t\right): t \in \mathbb{R}\right\} \subset \mathbb{C}$, has two-dimensional Lebesgue measure zero. Recently, Banks et. al [1] have shown that the curve $\left\{\zeta\left(\frac{1}{2}+i t\right): t \in \mathbb{R}\right\}$ has countably many selfintersections. From this, it immediately follows that there are only countably many numbers $a$ for which more than one $a$-point lies on the critical line. On the other hand, for every complex number $a$ we have from (1.3) that the Riemann hypothesis implies that no more than one-half of the $a$-points can lie on the line $\sigma=1 / 2$.

We will investigate the number of $a$-points that lie on the line $\sigma=1 / 2$ both unconditionally and under the assumption of a spacing condition on the ordinates of zeros of the Riemann zeta-function. Unconditionally, we show that for any nonzero complex number $a$ at most one-half of the nontrivial $a$-points of the Riemann zeta-function lie on the critical line. Furthermore, under the assumption of a spacing condition we prove that almost all of the $a$-points of $\zeta(s)$ do not lie on the line $\sigma=1 / 2$.

\section{Main Results}

Let us first introduce Hardy's Z-function in the form

$$
Z(t)=e^{i \theta(t)} \zeta\left(\frac{1}{2}+i t\right)
$$

where $\theta(t)$ is the Riemann-Siegel theta function and is given by

$$
\theta(t)=\arg \Gamma\left(\frac{1}{4}+i \frac{t}{2}\right)-\frac{\log \pi}{2} t
$$

Next, for any nonzero $a \in \mathbb{C}$ we write $a=|a| e^{i \phi}$ with $-\pi<\phi \leq \pi$ and let $g=g(\phi)$ be a solution to

$$
\theta(t) \equiv-\phi \quad(\bmod \pi)
$$

We call the points, $g$, shifted Gram points and for any $n \in \mathbb{Z}$ we call the unique $g=g_{n}$ that satisfies $\theta\left(g_{n}\right)=\pi n-\phi$ the $n$th shifted Gram point.

Let

$$
\Psi=\frac{1}{2} \log \log T \text {. }
$$

Also, write $\mathbf{1}_{S}(x)$ for the indicator function of the set $S$; that is $\mathbf{1}_{S}(x)$ equals one if $x \in S$ and equals zero otherwise. A result of Selberg states for $\alpha<\beta$ that

$$
\frac{1}{T} \int_{T}^{2 T} \mathbf{1}_{[\alpha, \beta]}\left(\log \left|\zeta\left(\frac{1}{2}+i t\right)\right| \Psi^{-1 / 2}\right) d t=\frac{1}{\sqrt{2 \pi}} \int_{\alpha}^{\beta} e^{-x^{2} / 2} d x+O\left(\frac{(\log \Psi)^{2}}{\sqrt{\Psi}}\right)
$$

(see [13] and [17]). Since shifted Gram points are regularly spaced (see Lemma 3.4) it seems reasonable to expect that $\log \left|\zeta\left(\frac{1}{2}+i g\right)\right| \Psi^{-1 / 2}$ is normally distributed at the shifted Gram points $T<g \leq 2 T$. In fact, Selberg, in unpublished work, proved that $\arg \zeta\left(\frac{1}{2}+i g\right) \Psi^{-1 / 2}$ has a normal limiting distribution.

However, there are extra considerations that need to be accounted for when estimating the real part of the logarithm. For instance, the possible existence of Landau-Siegel zeros could cause the ordinates of zeros of 
$\zeta(s)$ to be distributed according to the Alternative Hypothesis as stated in [6]. Hence, the presence of LandauSiegel zeros could imply that a positive proportion of the shifted Gram points $T<g \leq 2 T$ are equal to, or at least extremely close to, ordinates of zeros of $\zeta(s)$. This would show that $\log \left|\zeta\left(\frac{1}{2}+i g\right)\right| \Psi^{-1 / 2}$ is not normally distributed at these points.

For this reason we assume

Hypothesis S. For any $n \in \mathbb{N}$

$$
\lim _{\epsilon \rightarrow 0^{+}} \limsup _{T \rightarrow \infty} \frac{\left|\left\{T<\gamma, \gamma^{\prime} \leq 2 T:\left|\frac{\left(\gamma-\gamma^{\prime}\right) \log T}{2 \pi}-n\right|<\epsilon\right\}\right|}{T \log T}=0,
$$

where $\gamma, \gamma^{\prime}$ are ordinates of zeros of the Riemann zeta-function.

We note that this spacing hypothesis is similar to other spacing hypotheses made in [3], [7], and [4]. Additionally, we observe that if Montgomery's Pair Correlation Conjecture is true then so is Hypothesis S.

Montgomery's Pair Correlation Conjecture. For fixed $\alpha<\beta$ as $T \rightarrow \infty$

$$
\frac{1}{N(T)} \sum_{\substack{0<\gamma, \gamma^{\prime} \leq T \\ \frac{2 \pi \alpha}{\log T} \leq \gamma-\gamma^{\prime} \leq \frac{2 \pi \beta}{\log T}}} 1 \sim \int_{\alpha}^{\beta} 1-\left(\frac{\sin \pi x}{\pi x}\right)^{2} d x+\mathbf{1}_{[\alpha, \beta]}(0) .
$$

To calculate the distribution function of $\log \left|\zeta\left(\frac{1}{2}+i g\right)\right| \Psi^{-1 / 2}$ at the shifted Gram points $T<g \leq 2 T$ we will assume the truth of Hypothesis S. However, using the method of B. Hough [8] we can unconditionally establish an upper bound for the distribution function.

Theorem 2.1. For fixed $\alpha \in \mathbb{R}$ we have as $T \rightarrow \infty$

$$
\frac{1}{\frac{T}{2 \pi} \log T} \sum_{\substack{T<g \leq 2 T \\ g \neq \gamma}} \mathbf{1}_{[\alpha, \infty)}\left(\log \left|\zeta\left(\frac{1}{2}+i g\right)\right| \Psi^{-1 / 2}\right) \leq \frac{1}{\sqrt{2 \pi}} \int_{\alpha}^{\infty} e^{-x^{2} / 2} d x+o(1) .
$$

Assuming Hypothesis S we show the upper bound is best possible.

Theorem 2.2. Assume Hypothesis S. For fixed $\alpha<\beta$

$$
\lim _{T \rightarrow \infty} \frac{1}{\frac{T}{2 \pi} \log T} \sum_{\substack{T<g \leq 2 T \\ g \neq \gamma}} \mathbf{1}_{[\alpha, \beta]}\left(\log \left|\zeta\left(\frac{1}{2}+i g\right)\right| \Psi^{-1 / 2}\right)=\frac{1}{\sqrt{2 \pi}} \int_{\alpha}^{\beta} e^{-x^{2} / 2} d x .
$$

Establishing an upper bound on the rate of convergence to the Gaussian distribution in Theorem 2.2 would immediately lead to an improvement in Corollary 2.4. However, we are unable to do so. The limitation in our argument arises solely from Hypothesis S.

Recall that it follows from the work of Selberg (see [13] and [17]) that assuming the Riemann hypothesis at most one-half of the nontrivial $a$-points can lie on the critical line $\sigma=1 / 2$. From Theorem 2.1 we have that this holds unconditionally.

Corollary 2.3. For every nonzero complex number $a$ we have

$$
\frac{1}{N_{a}(T)} \sum_{\substack{0<\gamma_{a} \leq T \\ \beta_{a}=1 / 2}} 1 \leq \frac{1}{2}+o(1) \quad(T \rightarrow \infty) .
$$

That is, at most one-half of the nontrivial $a$-points of the Riemann zeta-function lie on the critical line.

Similarly, by Theorem 2.2 we have 
Corollary 2.4. Assume Hypothesis S. For every nonzero $a \in \mathbb{C}$, zero percent of the nontrivial $a$-points of $\zeta(s)$ lie on the critical line. That is,

$$
\lim _{T \rightarrow \infty} \frac{1}{N_{a}(T)} \sum_{\substack{0<\gamma_{a} \leq T \\ \beta_{a}=1 / 2}} 1=0
$$

By computing a mollified second moment of $\zeta\left(\frac{1}{2}+i g\right)-a$ one may be able to to give an alternative proof that a positive proportion of the $a$-points do not lie on the line $\sigma=1 / 2$. However, due the constraint on the length of the mollifier we believe that this would give an inferior result to Corollary 2.3.

As previously mentioned, an additional assumption on the zeros of $\zeta(s)$ is necessary for the conclusion of Theorem 2.2 to hold. We wonder if it is possible for the zeros of $\zeta(s)$ to be distributed in such a way so that a positive proportion of the nontrivial $a$-points lie on the line $\sigma=1 / 2$, for some $a \neq 0$. For instance, if the Alternative Hypothesis as stated in [6] is true then does the conclusion of Corollary 2.4 still hold? It is possible to give examples of functions $f(s)$ that are analytic in $0<\sigma<1$, whose zeros are regularly spaced, lie on the line $\sigma=1 / 2$, and for which many of the solutions to $f(s)=a$ also lie on the line $\sigma=1 / 2$. Simple examples of such functions are $f(s)=a \sinh \left(s-\frac{1}{2}\right)$ and $f(s)=\frac{|a|}{2} \chi(s)+\frac{a}{2}$ with $t>10$ (here $\chi$ is the functional equation factor). It would be interesting to determine what other functions also have these properties.

We now prove the corollaries.

Proof of Corollary 2.3. For any nonzero $a \in \mathbb{C}$ write $a=|a| e^{i \phi}$ with $-\pi<\phi \leq \pi$. Note that by the functional equation for $\zeta(s)$ it follows that $Z(t)$ is real. Thus, at an $a$-point of the form $\rho_{a}=1 / 2+i \gamma_{a}$, we have $Z\left(\gamma_{a}\right)=e^{i \theta\left(\gamma_{a}\right)} a=e^{i\left(\theta\left(\gamma_{a}\right)+\phi\right)}|a|$. Since $Z\left(\gamma_{a}\right)$ is real we have that

$$
\theta\left(\gamma_{a}\right) \equiv-\phi \quad(\bmod \pi)
$$

which implies that $\gamma_{a}$ is a shifted Gram point. Hence,

$$
\sum_{\substack{T<\gamma_{a} \leq 2 T \\ \beta_{a}=1 / 2}} 1 \leq \sum_{\substack{T<g \leq 2 T \\\left|\zeta\left(\frac{1}{2}+i g\right)\right|=|a|}} 1
$$

Next, let $A=|\log | a||$ and note that for any $\epsilon>0$, if $T$ is sufficiently large, then $2 A<\epsilon \sqrt{\Psi}$. Thus,

$$
\begin{aligned}
& \sum_{\substack{T<g \leq 2 T \\
\left|\zeta\left(\frac{1}{2}+i g\right)\right|=|a|}} 1 \leq \sum_{\substack{T<g \leq 2 T \\
g \neq \gamma}} \mathbf{1}_{[-2 A, 2 A]}\left(\log \left|\zeta\left(\frac{1}{2}+i g\right)\right|\right) \\
& \leq \sum_{\substack{T<g \leq 2 T \\
g \neq \gamma}} \mathbf{1}_{[-\epsilon, \epsilon]}\left(\log \left|\zeta\left(\frac{1}{2}+i g\right)\right| \Psi^{-1 / 2}\right) \\
& \leq \sum_{\substack{T<g \leq 2 T \\
g \neq \gamma}} \mathbf{1}_{[-\epsilon, \infty)}\left(\log \left|\zeta\left(\frac{1}{2}+i g\right)\right| \Psi^{-1 / 2}\right) .
\end{aligned}
$$

Observe that by Theorem 2.1 we have

$$
\frac{1}{\frac{T}{2 \pi} \log T} \sum_{\substack{T<g \leq 2 T \\ g \neq \gamma}} \mathbf{1}_{[-\epsilon, \infty)}\left(\log \left|\zeta\left(\frac{1}{2}+i g\right)\right| \Psi^{-1 / 2}\right) \leq \frac{1}{\sqrt{2 \pi}} \int_{-\varepsilon}^{\infty} e^{-x^{2} / 2} d x+o(1)=\frac{1}{2}+o(1)
$$

since $\epsilon>0$ is arbitrary. Combining this with (2.1) and (2.2), we have

$$
\sum_{\substack{T<\gamma_{a} \leq 2 T \\ \beta_{a}=1 / 2}} 1 \leq\left(\frac{1}{2}+o(1)\right) \frac{T}{2 \pi} \log T
$$

For any positive integer $N$ observe that we have

$$
\sum_{\substack{T \\ 2^{N}<\gamma_{a} \leq T \\ \beta_{a}=1 / 2}} 1=\sum_{k=0}^{N-1} \sum_{\substack{\frac{T}{2^{k+1}<\gamma_{a} \leq \frac{T}{2^{k}}} \\ \beta_{a}=1 / 2}} 1 \leq\left(\frac{1}{2}+o(1)\right)\left(\sum_{k=0}^{N-1} \frac{T}{2^{k+1}(2 \pi)} \log \frac{T}{2^{k+1}}\right)=\left(\frac{1}{2}+o(1)\right) \frac{T}{2 \pi} \log T
$$


since we may take $N$ arbitrarily large. Recall that

$$
N_{a}(T)=\sum_{1<\gamma_{a} \leq T} 1=\frac{T}{2 \pi} \log \frac{T}{2 \pi}+O(T) .
$$

From this we see that

$$
\sum_{\substack{0<\gamma_{a} \leq \frac{T}{2^{N}} \\ \beta_{a}=1 / 2}} 1 \leq N_{a}\left(T / 2^{N}\right) \ll \frac{T}{2^{N}} \log T=o(T \log T) .
$$

Corollary 2.3 now follows.

Proof of Corollary 2.4. The argument is similar to the previous proof. Assuming Hypothesis $\mathrm{S}$ we have by Theorem 2.2 for any fixed $\epsilon>0$ that as $T \rightarrow \infty$

$$
\sum_{\substack{T<g \leq 2 T \\ g \neq \gamma}} \mathbf{1}_{[-\epsilon, \epsilon]}\left(\log \left|\zeta\left(\frac{1}{2}+i g\right)\right| \Psi^{-1 / 2}\right)=\frac{T \log T}{(2 \pi)^{3 / 2}}\left(\int_{-\epsilon}^{\epsilon} e^{-x^{2} / 2} d x+o(1)\right)=o(T \log T)
$$

since $\epsilon$ was arbitrary. Using this estimate in (2.2) we have by (2.1) that

$$
\sum_{\substack{T<\gamma_{a} \leq 2 T \\ \beta_{a}=1 / 2}} 1=o(T \log T)
$$

The proof is completed along the same lines as before.

\section{Preliminary Lemmas}

The following estimate is due to van der Corput and can be found in [16].

Lemma 3.1. Suppose that $f(u)$ is real and twice differentiable and that $f^{\prime \prime}(u) \approx \lambda$ on an interval $[a, b]$ with $b \geq a+1$. Then

$$
\sum_{a<n \leq b} \exp (2 \pi i f(n)) \ll(b-a) \lambda^{1 / 2}+\lambda^{-1 / 2} .
$$

Lemma 3.2. Let $T \geq 10$. Then for positive $x$ not equal to one

$$
\sum_{T<g \leq 2 T} x^{i g} \ll\left(T \frac{|\log x|}{\log T}\right)^{1 / 2}+\left(T \frac{\log ^{3} T}{|\log x|}\right)^{1 / 2}+|\log x| .
$$

Proof. Our first step will be to derive an approximate formula for $g_{n}$. By Stirling's formula, for $t>1$,

$$
\frac{\theta(t)}{\pi}+\frac{\phi}{\pi}=\frac{t}{2 \pi} \log \frac{t}{2 \pi e}-\frac{1}{8}+\frac{\phi}{\pi}+O(1 / t) .
$$

We now let $\tilde{g}_{n}$ be defined via the equation

$$
\frac{\tilde{g}_{n}}{2 \pi} \log \frac{\tilde{g}_{n}}{2 \pi e}-\frac{1}{8}+\frac{\phi}{\pi}=n
$$

Next write $Z=\log \left(\tilde{g}_{n} /(2 \pi e)\right)$ so that

$$
\frac{n}{e}+\frac{1}{8 e}-\frac{\phi}{\pi e}=Z e^{Z} .
$$

Writing, $W(Z)$ for the Lambert $W$-function, which is the inverse function of $Z e^{Z}$, we have $W\left(\frac{n}{e}+\frac{1}{8 e}-\frac{\phi}{\pi e}\right)=$ $Z=\log \left(\tilde{g}_{n} /(2 \pi e)\right)$, so that

$$
\tilde{g}_{n}=2 \pi \exp \left(1+W\left(\frac{n}{e}+\frac{1}{8 e}-\frac{\phi}{\pi e}\right)\right)
$$


Now suppose that $n>(\theta(T)+\phi) / \pi$, so that $g_{n}>T$. By definition,

$$
\left(\frac{\tilde{g}_{n}}{2 \pi} \log \frac{\tilde{g}_{n}}{2 \pi e}-\frac{1}{8}+\frac{\phi}{\pi}\right)-\left(\frac{\theta\left(g_{n}\right)}{\pi}+\frac{\phi}{\pi}\right)=n-n=0
$$

and by (3.1),

$$
\frac{\theta\left(g_{n}\right)}{\pi}=\frac{g_{n}}{2 \pi} \log \frac{g_{n}}{2 \pi e}-\frac{1}{8}+O(1 / T) .
$$

Consequently,

$$
\left|\frac{g_{n}}{2 \pi} \log \frac{g_{n}}{2 \pi e}-\frac{\tilde{g}_{n}}{2 \pi} \log \frac{\tilde{g}_{n}}{2 \pi e}\right| \ll 1 / T .
$$

Next consider the function $f(x)=x \log (x /(2 \pi e))$. For any $x>y \geq 10$

$$
\int_{y}^{x} f^{\prime}(t) d t \geq(x-y) \log \frac{y}{2 \pi}
$$

Hence, if $f(x)-f(y) \leq X$ then $x-y \leq X / \log (y / 2 \pi)$. Thus, for $n>(\theta(T)+\phi) / \pi$ we have $g_{n}=\tilde{g}_{n}+$ $O(1 /(T \log T))$. Let $A(t)=(\theta(t)+\phi) / \pi$ and note that for real $a, b$ we have $|\exp (i b)-\exp (i a)|=\left|\int_{a}^{b} \exp (i t) d t\right| \leq$ $|b-a|$. Therefore,

$$
\sum_{T<g \leq 2 T} x^{i g}=\sum_{A(T)<n \leq A(2 T)} x^{i g_{n}}=\sum_{A(T)<n \leq A(2 T)} x^{i \tilde{g}_{n}}+O(|\log x|) .
$$

Let $F(u)=\log x \exp \left(1+W\left(\frac{u}{e}+\frac{1}{8 e}-\frac{\phi}{\pi e}\right)\right)$ and observe that $\exp (2 \pi i F(n))=x^{i \tilde{g}_{n}}$. Note that

$$
W^{\prime}(u)=\frac{1}{\exp (W(u))(1+W(u))}=\frac{W(u)}{u(1+W(u))},
$$

and $W(u) \approx \log u$. So that for $u \geq 10$

$$
F^{\prime \prime}(u)=\frac{-\log x W\left(\frac{u}{e}+\frac{1}{8 e}-\frac{\phi}{\pi e}\right)}{\left(u+\frac{1}{8}-\frac{\phi}{\pi}\right)\left(1+W\left(\frac{u}{e}+\frac{1}{8 e}-\frac{\phi}{\pi e}\right)\right)^{3}} \approx \frac{|\log x|}{u \log ^{2} u} .
$$

We take $a=A(T), b=A(2 T), f(u)=F(u)$, and $\lambda=|\log x| /\left(T \log ^{3} T\right)$. The result now follows from applying Lemma 3.1 to the sum on the right-hand side of (3.2).

Let

$$
N_{g}(T)=\sum_{0<g \leq T} 1=\frac{T}{2 \pi} \log \frac{T}{2 \pi e}+O(1) \quad \text { and } \quad N_{g}(T, 2 T)=N_{g}(2 T)-N_{g}(T) .
$$

Lemma 3.3. Let $2 \leq x \leq T^{1 / 4}$ and for each prime $p$ let $a_{p}$ be a complex number. If

$$
a_{p} \ll \frac{\log p}{p^{1 / 2} \log x},
$$

then

$$
\sum_{T<g \leq 2 T}\left|\sum_{p \leq x} a_{p} p^{-i g}\right|^{2} \ll T \log T
$$

Also, if

$$
a_{p} \ll 1
$$

then

$$
\sum_{T<g \leq 2 T}\left|\sum_{p \leq x} \frac{a_{p}}{p^{1+2 i g}}\right|^{2} \ll T \log T
$$


Proof. We begin by proving the first assertion. By Lemma 3.2,

$$
\begin{aligned}
\sum_{T<g \leq 2 T}\left|\sum_{p \leq x} a_{p} p^{-i g}\right|^{2} & =N_{g}(T, 2 T) \sum_{p \leq x}\left|a_{p}\right|^{2}+\sum_{\substack{p, q \leq x \\
p \neq q}} a_{p} \bar{a}_{q} \sum_{T<g \leq 2 T}\left(\frac{p}{q}\right)^{i g} \\
& \ll T \frac{\log T}{\log ^{2} x} \sum_{p \leq x} \frac{\log ^{2} p}{p}+T^{1 / 2} \frac{(\log T)^{3 / 2}}{\log ^{2} x} \sum_{\substack{p, q \leq x \\
p \neq q}} \frac{\log p \log q}{\sqrt{p q\left|\log \frac{p}{q}\right|}} .
\end{aligned}
$$

Since

$$
\sum_{p \leq x} \frac{\log ^{2} p}{p} \ll \log ^{2} x
$$

the first term above is $\ll T \log T$. To estimate the second term on the right-hand side, observe that if $p<q \leq x$

$$
\log q-\log p=\int_{p}^{q} \frac{d t}{t} \geq \frac{q-p}{p} \geq \frac{1}{x} .
$$

Hence,

$$
\sum_{\substack{p, q \leq x \\ p \neq q}} \frac{\log p \log q}{\sqrt{p q\left|\log \frac{p}{q}\right|}} \ll x^{1 / 2}\left(\sum_{p \leq x} \frac{\log p}{\sqrt{p}}\right)^{2} \ll x^{3 / 2}
$$

So that

$$
T^{1 / 2} \frac{(\log T)^{3 / 2}}{\log ^{2} x} \sum_{\substack{p, q \leq x \\ p \neq q}} \frac{\log p \log q}{\sqrt{p q\left|\log \frac{p}{q}\right|}} \ll T^{1 / 2} x^{3 / 2} \frac{(\log T)^{3 / 2}}{\log ^{2} x} \ll T .
$$

Applying this in (3.5) yields the first assertion of the lemma.

As for the second assertion we argue similarly to obtain

$$
\sum_{T<g \leq 2 T}\left|\sum_{p \leq x} \frac{a_{p}}{p^{1+2 i g}}\right|^{2} \ll T \log T \sum_{p \leq x} \frac{1}{p^{2}}+T^{1 / 2}(\log T)^{3 / 2} \sum_{\substack{p, q \leq x \\ p \neq q}} \frac{1}{p q \sqrt{\left|\log \frac{p}{q}\right|}},
$$

which, as before, is seen to be $\ll T \log T$.

Next, we have

Lemma 3.4. For any $m, \ell \in \mathbb{N}$ satisfying $N_{g}(T)<m<\ell \leq N_{g}(2 T)$, we have

$$
\left(g_{\ell}-g_{m}\right) \frac{\log T}{2 \pi}=(\ell-m)(1+O(1 / \log T)) .
$$

Proof. By Stirling's formula, for $N_{g}(T)<\ell \leq N_{g}(2 T)$

$$
\ell=\frac{\theta\left(g_{\ell}\right)}{\pi}+\frac{\phi}{\pi}=\frac{g_{\ell}}{2 \pi}(\log T+O(1)) .
$$

Differencing this with the analogous formula for $m$, we obtain

$$
(\ell-m)=\left(g_{\ell}-g_{m}\right) \frac{\log T}{2 \pi}(1+O(1 / \log T)) .
$$

The next lemma is from K. M. Tsang's PhD thesis [17] and follows from the zero density estimate

$$
\sum_{\substack{0<\gamma \leq T \\ \beta>\sigma}} 1 \ll T^{1-(\sigma-1 / 2) / 4} \log T
$$

due to Selberg [12]. 
Lemma 3.5. Let $3 \leq \xi \leq T^{1 / 8}$. For $k \geq 0$ and $1 / 2 \leq \sigma \leq 1$, we have

$$
\sum_{\substack{0<\gamma \leq T \\ \beta>\sigma}}(\beta-\sigma)^{k} \xi^{\beta-\sigma} \ll T^{1-(\sigma-1 / 2) / 4}(\log T)^{1-k},
$$

where the implied constant depends on $k$.

For $X>0$ and $t \geq 2$ we define the number

$$
\sigma_{X, t}=\frac{1}{2}+2 \max \left(\beta-\frac{1}{2}, \frac{2}{\log X}\right)
$$

where the maximum is taken over $\rho$ satisfying $|t-\gamma| \leq X^{3|\beta-1 / 2|} / \log X$.

Lemma 3.6. Let $3 \leq \xi \leq T^{1 / 25}$ and $X=T^{1 / 100}$. Then for $k \geq 0$ we have

$$
\sum_{T<g \leq 2 T}\left(\sigma_{X, g}-\frac{1}{2}\right)^{k} \xi^{\sigma_{X, g}-1 / 2} \ll T \log ^{1-k} T
$$

where the implied constant depends on $k$.

Proof. By the definition of $\sigma_{X, t}$, if for some $g$ we have $\sigma_{X, g}>1 / 2+4 / \log X$ then there is a $\rho_{0}$ such that $\beta_{0}>1 / 2+2 / \log X$ and $\left|g-\gamma_{0}\right| \leq X^{3(\beta-1 / 2)} / \log X$. In particular, if $T<g \leq 2 T$, then $0<\gamma_{0} \leq 3 T$. Let

$$
G(\beta+i \gamma)=\left|\left\{T<g \leq 2 T:|g-\gamma| \leq \frac{X^{3(\beta-1 / 2)}}{\log X}\right\}\right| .
$$

Then

$$
\sum_{T<g \leq 2 T}\left(\sigma_{X, g}-\frac{1}{2}\right)^{k} \xi^{\sigma_{X, g}-1 / 2} \ll \sum_{T<g \leq 2 T} \frac{1}{\log ^{k} X}+\sum_{\substack{0<\gamma \leq 3 T \\ \beta>1 / 2+\frac{2}{\log X}}}\left(\beta-\frac{1}{2}\right)^{k} \xi^{2(\beta-1 / 2)} G(\beta+i \gamma) .
$$

For any zero of $\zeta(s)$, we have that

$$
G(\beta+i \gamma) \ll \frac{X^{3(\beta-1 / 2)} \log T}{\log X},
$$

because the points $g$ are regularly spaced approximately $(\log T)^{-1}$ apart. Hence, the right-hand side of (3.7) is

$$
\ll T \frac{\log T}{\log ^{k} X}+\frac{\log T}{\log X} \sum_{\substack{0<\gamma \leq 3 T \\ \beta>1 / 2}}\left(\beta-\frac{1}{2}\right)^{k}\left(\xi^{2} X^{3}\right)^{\beta-1 / 2} .
$$

Applying Lemma 3.5, we see that both terms are

$$
\ll T \log ^{1-k} T .
$$

Lemma 3.7. Let $m \in \mathbb{N}$ and $2 \leq Y \leq T^{1 / m}$. Then

$$
\frac{1}{T} \int_{0}^{T}\left(\sum_{p \leq Y} \frac{\cos (t \log p)}{p^{1 / 2}}\right)^{m} d t=\int_{[0,1]^{\pi(Y)}}\left(\sum_{p \leq Y} \frac{\cos \left(2 \pi \theta_{p}\right)}{p^{1 / 2}}\right)^{m} d \theta+O\left(T^{-1 / 2}(c m)^{m / 2}\right),
$$

where $d \theta=\prod_{p \leq Y} d \theta_{p}$ and $c$ is an absolute constant. If $m=0$ this holds without the error term. Furthermore, for $m \in \mathbb{N}$ we have

$$
\frac{1}{T} \int_{0}^{T}\left|\sum_{p \leq Y} \frac{\cos (t \log p)}{p^{1 / 2}}\right|^{m} d t, \quad \int_{[0,1]^{\pi(Y)}}\left|\sum_{p \leq Y} \frac{\cos \left(2 \pi \theta_{p}\right)}{p^{1 / 2}}\right|^{m} d \theta \ll(\Psi c m)^{m / 2},
$$

where $c$ is a positive absolute constant. 
Proof. This is essentially Lemma 3.4 of [17].

Lemma 3.8. Let $Y=T^{1 / \Psi^{4}}$. For $|u| \leq \Psi^{2}$ we have

$$
\begin{aligned}
\frac{1}{T} \int_{T}^{2 T} \exp \left(i u \sum_{p \leq Y} \frac{\cos (t \log p)}{p^{1 / 2}} \Psi^{-1 / 2}\right) d t= & \int_{[0,1] \pi(Y)} \exp \left(i u \sum_{p \leq Y} \frac{\cos \left(2 \pi \theta_{p}\right)}{p^{1 / 2}} \Psi^{-1 / 2}\right) d \theta \\
& +O\left(|u| e^{-\Psi^{5}}\right)
\end{aligned}
$$

where $d \theta=\prod_{p \leq y} d \theta_{p}$.

Proof. We expand the exponential function to see that

$$
\begin{aligned}
\frac{1}{T} \int_{T}^{2 T} \exp \left(i u \sum_{p \leq Y} \frac{\cos (t \log p)}{p^{1 / 2}} \Psi^{-1 / 2}\right) d t= & \sum_{n=0}^{N-1} \frac{\left(i u \Psi^{-1 / 2}\right)^{n}}{n !} \frac{1}{T} \int_{T}^{2 T}\left(\sum_{p \leq Y} \frac{\cos (t \log p)}{p^{1 / 2}}\right)^{n} d t \\
& +O\left(\frac{\left|u \Psi^{-1 / 2}\right|^{N}}{N !} \frac{1}{T} \int_{T}^{2 T}\left|\sum_{p \leq Y} \frac{\cos (t \log p)}{p^{1 / 2}}\right|^{N} d t\right) .
\end{aligned}
$$

By Lemma 3.7 we obtain

$$
\begin{aligned}
\frac{1}{T} \int_{T}^{2 T} \exp \left(i u \sum_{p \leq Y} \frac{\cos (t \log p)}{p^{1 / 2}} \Psi^{-1 / 2}\right) d t= & \sum_{n=0}^{N-1} \frac{\left(i u \Psi^{-1 / 2}\right)^{n}}{n !} \int_{[0,1]^{\pi(Y)}}\left(\sum_{p \leq Y} \frac{\cos \left(2 \pi \theta_{p}\right)}{p^{1 / 2}}\right)^{n} d \theta \\
& +O\left(\frac{|u|^{N}(c N)^{N / 2}}{N !}\right)+O\left(T^{-1 / 2} \sum_{n=1}^{N-1} \frac{|u|^{n}(c n)^{n / 2}}{n !}\right) .
\end{aligned}
$$

This equals

$$
\int_{[0,1]^{\pi(Y)}} \exp \left(i u \sum_{p \leq Y} \frac{\cos \left(2 \pi \theta_{p}\right)}{p^{1 / 2}} \Psi^{-1 / 2}\right) d \theta+O\left(\frac{|u|^{N}(c N)^{N / 2}}{N !}\right)+O\left(T^{-1 / 2} \sum_{n=1}^{N-1} \frac{|u|^{n}(c n)^{n / 2}}{n !}\right) .
$$

We now take $N=2\left\lfloor\Psi^{5}\right\rfloor$ so that $Y \leq T^{1 / N}$, and note that $|u| \leq \Psi^{2}$. We then find that

$$
\frac{|u|^{N}(c N)^{N / 2}}{N !} \ll|u| \frac{\Psi^{2 N-2}(c N)^{N / 2}}{N !} \ll|u|\left(c N^{-1} \Psi^{4}\right)^{N / 2} \ll|u| e^{-\Psi^{5}},
$$

by Stirling's formula. The other $O$-term is estimated along the same lines.

Lemma 3.9. Let $X=T^{1 / 100}$. For $|u| \leq \Psi^{1 / 2} / 100$, we have

$$
\begin{aligned}
\frac{1}{T} \int_{T}^{2 T} \exp \left(i u \sum_{p \leq X^{3}} \frac{\cos (t \log p)}{p^{1 / 2}} \Psi^{-1 / 2}\right) d t= & e^{-u^{2} / 2}\left(1+O\left(\frac{u^{2} \log \log \log T}{\Psi}\right)\right) \\
& +O\left(|u|\left(\frac{\log \log \log T}{\Psi}\right)^{1 / 2}\right) .
\end{aligned}
$$

Proof. For real $u, v$, we have $|\exp (i u)-\exp (i v)|=\left|\int_{v}^{u} \exp (i t) d t\right| \leq|u-v|$. Hence, letting $Y=T^{1 / \Psi^{4}}$, we find that

$$
\frac{1}{T} \int_{T}^{2 T} \exp \left(i u \sum_{p \leq X^{3}} \frac{\cos (t \log p)}{p^{1 / 2}} \Psi^{-1 / 2}\right) d t
$$

equals

$$
\frac{1}{T} \int_{T}^{2 T} \exp \left(i u \sum_{p \leq Y} \frac{\cos (t \log p)}{p^{1 / 2}} \Psi^{-1 / 2}\right) d t+O\left(\frac{|u|}{\Psi^{1 / 2}} \frac{1}{T} \int_{T}^{2 T}\left|\sum_{Y<p \leq X^{3}} \frac{\cos (t \log p)}{p^{1 / 2}}\right| d t\right) .
$$


To estimate the integral in the error term we apply Cauchy's inequality and Montgomery and Vaughan's mean value theorem for Dirichlet polynomials [11] to see that

$$
\begin{aligned}
\int_{T}^{2 T}\left|\sum_{Y<p \leq X^{3}} \frac{\cos (t \log p)}{p^{1 / 2}}\right| d t & \ll T\left(\sum_{Y<p \leq X^{3}} \frac{1}{p}\right)^{1 / 2} \\
& \ll T\left(\log \frac{3 \log X}{\log Y}\right)^{1 / 2} \\
& \ll T(\log \log \log T)^{1 / 2}
\end{aligned}
$$

Thus, by this and Lemma 3.8 it follows that

$$
\begin{aligned}
\frac{1}{T} \int_{T}^{2 T} \exp \left(i u \sum_{p \leq X^{3}} \frac{\cos (t \log p)}{p^{1 / 2}} \Psi^{-1 / 2}\right) d t= & \int_{[0,1]^{\pi(Y)}} \exp \left(i u \sum_{p \leq Y} \frac{\cos \left(2 \pi \theta_{p}\right)}{p^{1 / 2}} \Psi^{-1 / 2}\right) d \theta \\
& +O\left(|u| e^{-\Psi^{5}}\right)+O\left(|u|\left(\frac{\log \log \log T}{\Psi}\right)^{1 / 2}\right)
\end{aligned}
$$

Next, observe that

$$
\begin{aligned}
\int_{[0,1]^{\pi(Y)}} \exp \left(i u \sum_{p \leq Y} \frac{\cos \left(2 \pi \theta_{p}\right)}{p^{1 / 2}} \Psi^{-1 / 2}\right) d \theta & =\prod_{p \leq Y} \int_{0}^{1} \exp \left(i u \frac{\cos \left(2 \pi \theta_{p}\right)}{p^{1 / 2}} \Psi^{-1 / 2}\right) d \theta_{p} \\
& =\prod_{p \leq Y} J_{0}\left(\frac{u}{(p \Psi)^{1 / 2}}\right)
\end{aligned}
$$

where $J_{0}(z)=\int_{0}^{1} e^{i z \cos (2 \pi \theta)} d \theta$ is the zeroth Bessel function of the first kind. This function also has the series expansion

$$
J_{0}(z)=\sum_{n=0}^{\infty}(-1)^{n} \frac{\left(\frac{1}{2} z\right)^{2 n}}{(n !)^{2}} .
$$

Consequently, for $|z| \leq 1$ we have

$$
J_{0}(2 z)=e^{-z^{2}}\left(1+O\left(|z|^{4}\right)\right)
$$

It follows that

$$
\prod_{p \leq Y} J_{0}\left(\frac{u}{(p \Psi)^{1 / 2}}\right)=\exp \left(-\frac{u^{2}}{4 \Psi} \sum_{p \leq Y} \frac{1}{p}\right) \prod_{p \leq Y}\left(1+O\left(\frac{u^{4}}{p^{2} \Psi^{2}}\right)\right) .
$$

A simple calculation shows that $\prod_{p \leq Y}\left(1+O\left(u^{4} /\left(p^{2} \Psi^{2}\right)\right)\right)=1+O\left(u^{4} / \Psi^{2}\right)$. Next, note that

$$
\frac{1}{\Psi} \sum_{p \leq Y} \frac{1}{p}=2 \frac{\log \log Y+O(1)}{\log \log T+O(1)}=2(1+O(\log \log \log T / \log \log T)) .
$$

Thus, for $|u| \leq \Psi^{1 / 2} / 100$, we have

$$
\exp \left(-\frac{u^{2}}{4 \Psi} \sum_{p \leq Y} \frac{1}{p}\right) \prod_{p \leq Y}\left(1+O\left(\frac{u^{4}}{p^{2} \Psi^{2}}\right)\right)=\exp \left(-u^{2} / 2\right)\left(1+O\left(\frac{u^{2} \log \log \log T}{\log \log T}\right)\right) .
$$

Therefore, by this, (3.8), (3.9), and (3.10) the result follows.

\section{An Approximate Formula for $\log \left|\zeta\left(\frac{1}{2}+i t\right)\right|$}

In [12] Selberg proves an explicit formula for $S(t)=\pi^{-1} \arg \zeta\left(\frac{1}{2}+i t\right)$ in terms of a Dirichlet polynomial supported on prime numbers. The purpose of this section is to prove an analogous formula for $\log \left|\zeta\left(\frac{1}{2}+i t\right)\right|$.

For any real number $t$, let

$$
\eta_{t}=\min _{\gamma}|t-\gamma|
$$


For $x \geq 2$ define

$$
w_{x}(n)= \begin{cases}1 & \text { if } n \leq x, \\ \frac{\log ^{2}\left(x^{3} / n\right)-2 \log ^{2}\left(x^{2} / n\right)}{2 \log ^{2} x} & \text { if } x<n \leq x^{2}, \\ \frac{\log ^{2}\left(x^{3} / n\right)}{2 \log ^{2} x} & \text { if } x^{2}<n \leq x^{3} \\ 0 & \text { if } n>x^{3}\end{cases}
$$

Next, write $\log ^{+} x$ for the positive part of the logarithm, that is $\log ^{+} x=\log x$ if $x>1$ and $\log ^{+} x=0$ for $0<x \leq 1$. Now, let

$$
F(t ; X)=\left(\frac{X^{\left(\frac{1}{2}-\sigma_{X, t}\right) / 2}}{\log X}+\left(\sigma_{X, t}-\frac{1}{2}\right)\right)\left(\left(\sigma_{X, t}-\frac{1}{2}\right) \log X+\log ^{+} \frac{1}{\eta_{t} \log X}\right)
$$

where $\sigma_{X, t}$ is defined in (3.6). Also, let

$$
E_{1}(t ; X)=\left|\sum_{n \leq X^{3}} \frac{\Lambda(n)}{n^{\sigma}(n, t+i t} w_{X}(n)\right| .
$$

We now cite

Lemma 4.1. For $T<t \leq 2 T$ and $2 \leq X \leq T^{1 / 100}$ we have

$$
\log \left|\zeta\left(\frac{1}{2}+i t\right)\right|=\sum_{n \leq X^{3}} \frac{\Lambda(n) \cos (t \log n)}{n^{\sigma_{X, t}} \log n} w_{X}(n)+O\left(F(t ; X)\left(E_{1}(t ; X)+\log T\right)\right) .
$$

Additionally, under the same hypotheses

$$
\log \left|\zeta\left(\sigma_{X, t}+i t\right)\right|=\sum_{n \leq X^{3}} \frac{\Lambda(n) \cos (t \log n)}{n^{\sigma_{X, t}} \log n} w_{X}(n)+O\left(\frac{X^{\left(\frac{1}{2}-\sigma_{X, t}\right) / 2}}{\log X}\left(E_{1}(t ; X)+\log T\right)\right) .
$$

Proof. The first statement is proved in K. M. Tsang's PhD thesis (see Theorem 5.2 of [17]). The second formula is due to A. Selberg (see equation (4.9) of [12]).

Lemma 4.2. For $T<t \leq 2 T$ and $2 \leq X \leq T^{1 / 100}$ we have

$$
\begin{aligned}
\log \left|\zeta\left(\frac{1}{2}+i t\right)\right|= & \sum_{p \leq X^{3}} \frac{\cos (t \log p)}{p^{1 / 2}}+O\left(F(t ; X) \log T+E_{2}(t ; X)+E_{3}(t ; X)\right) \\
& +O\left(F(t ; X) X^{\sigma_{X, t}-\frac{1}{2}} \int_{1 / 2}^{\infty} X^{1 / 2-u}\left|\sum_{n \leq X^{3}} \frac{\log p \log p X}{p^{u+i t}} w_{X}(p)\right| d u\right)
\end{aligned}
$$

where $F(t ; T)$ is defined in $(4.1)$ and

$$
E_{2}(t ; X)=\left|\sum_{p \leq X^{3}} \frac{\left(1-w_{X}(p)\right)}{p^{1 / 2+i t}}\right| \quad \text { and } \quad E_{3}(t ; X)=\left|\sum_{p \leq X^{3 / 2}} \frac{w_{X}\left(p^{2}\right)}{p^{1+2 i t}}\right| .
$$

Additionally, under the same hypotheses

$$
\begin{aligned}
\log \left|\zeta\left(\sigma_{X, t}+i t\right)\right|= & \sum_{p \leq X^{3}} \frac{\cos (t \log p)}{p^{1 / 2}}+O\left(\left(\frac{X^{\left(\frac{1}{2}-\sigma_{X, t}\right) / 2}}{\log X}+\left(\sigma_{X, t}-\frac{1}{2}\right)\right) \log T+E_{2}(t ; X)+E_{3}(t ; X)\right) \\
& +O\left(\left(\frac{X^{\left(\sigma_{X, t}-\frac{1}{2}\right) / 2}}{\log X}+\left(\sigma_{X, t}-\frac{1}{2}\right) X^{\sigma_{X, t}-\frac{1}{2}}\right) \int_{1 / 2}^{\infty} X^{1 / 2-u}\left|\sum_{n \leq X^{3}} \frac{\log p \log p X}{p^{u+i t}} w_{X}(p)\right| d u\right) .
\end{aligned}
$$


Proof. We prove only the first assertion. The second statement follows from essentially the same argument.

From Lemma 4.1 we have that

$$
\log \left|\zeta\left(\frac{1}{2}+i t\right)\right|=\sum_{n \leq X^{3}} \frac{\Lambda(n) \cos (t \log n)}{n^{\sigma, t,} \log n} w_{X}(n)+O\left(F(t ; X)\left(E_{1}(t ; X)+\log T\right)\right)
$$

We now split the sum into a sum over primes, a sum over squares of primes, and a sum over the higher prime powers. In the sum over primes we replace the weight $w_{X}(n)$ with 1 and $\sigma_{X, t}$ with $1 / 2$. For the sum over squares of primes we replace $\sigma_{X, t}$ with $1 / 2$, and the sum over the higher prime powers is estimated trivially. We also use the inequality $|\Re z| \leq|z|$. In this way we find that

$$
\begin{aligned}
\sum_{n \leq X^{3}} \frac{\Lambda(n) \cos (t \log n)}{n^{\sigma}, t \log n} w_{X}(n)= & \sum_{p \leq X^{3}} \frac{\cos (t \log p)}{p^{1 / 2}}+O\left(\left|\sum_{p \leq X^{3}} \frac{\left(1-w_{X}(p)\right)}{p^{1 / 2+i t}}\right|\right) \\
& +O\left(\left|\sum_{p \leq X^{3}} w_{X}(p) p^{-i t}\left(p^{-\sigma_{X, t}}-p^{-1 / 2}\right)\right|\right)+O\left(\left|\sum_{p \leq X^{3 / 2}} \frac{w_{X}\left(p^{2}\right)}{p^{1+2 i t}}\right|\right) \\
& +O\left(\left|\sum_{p \leq X^{3 / 2}} w_{X}\left(p^{2}\right) p^{-2 i t}\left(p^{-2 \sigma_{X, t}}-p^{-1}\right)\right|\right)+O\left(\sum_{\substack{p^{r} \leq X^{3} \\
r>2}} \frac{1}{r p^{r / 2}}\right) .
\end{aligned}
$$

The first $O$-term is $\ll E_{2}(t ; X)$ and the third $O$-term is $\ll E_{3}(t ; X)$. Next, observe that

$$
\sum_{\substack{r \\ p^{r}>X^{3} \\ r>2}} \frac{1}{r p^{r / 2}} \ll 1 \ll\left(\sigma_{X, t}-\frac{1}{2}\right) \log T
$$

so the last error term in (4.3) is bound by $F(t ; X) \log T$. To bound the fourth $O$-term note that

$$
\begin{aligned}
\sum_{p \leq X^{3 / 2}} w_{X}\left(p^{2}\right) p^{-2 i t}\left(p^{-2 \sigma_{X, t}}-p^{-1}\right) & \ll \sum_{p \leq X^{3 / 2}} \frac{1-p^{1-2 \sigma_{X, t}}}{p} \\
& \ll\left(\sigma_{X, t}-\frac{1}{2}\right) \sum_{p \leq X^{3 / 2}} \frac{\log p}{p} \\
& \ll\left(\sigma_{X, t}-\frac{1}{2}\right) \log T,
\end{aligned}
$$

which again is $\ll F(t ; X) \log T$.

To complete the proof there are two things to show. First, that the second error term in (4.3) is bounded by

$$
F(t ; X) X^{\sigma_{X, t}-1 / 2} \int_{1 / 2}^{\infty} X^{1 / 2-u}\left|\sum_{p \leq X^{3}} \frac{\log p \log p X}{p^{u+i t}} w_{X}(p)\right| d u .
$$

The second thing to show is that $F(t ; X) E_{1}(t ; X)$ is bounded by (4.4) plus $F(t ; X) \log T$.

To begin, observe that

$$
\begin{aligned}
\sum_{p \leq X^{3}} \frac{w_{X}(p)}{p^{i t}}\left(p^{-\sigma_{X, t}}-p^{-1 / 2}\right) & =\int_{1 / 2}^{\sigma_{X, t}} \sum_{p \leq X^{3}} \frac{\log p}{p^{u+i t}} w_{X}(p) d u \\
& \ll\left(\sigma_{X, t}-\frac{1}{2}\right)\left|\sum_{p \leq X^{3}} \frac{\log p}{p^{\sigma^{*}+i t}} w_{X}(p)\right|,
\end{aligned}
$$

where $\sigma^{*}=\sigma^{*}(t)$ lies between $1 / 2$ and $\sigma_{X, t}$. Next, we see that if $1 / 2 \leq \sigma \leq \sigma_{X, t}$, then

$$
\begin{aligned}
\left|\sum_{p \leq X^{3}} \frac{\log p}{p^{\sigma+i t}} w_{X}(p)\right| & =\left|X^{\sigma-1 / 2} \int_{\sigma}^{\infty} X^{1 / 2-u} \sum_{p \leq X^{3}} \frac{\log p \log X p}{p^{u+i t}} w_{X}(p) d u\right| \\
& \leq X^{\sigma_{X, t}-1 / 2} \int_{1 / 2}^{\infty} X^{1 / 2-u}\left|\sum_{p \leq X^{3}} \frac{\log p \log X p}{p^{u+i t}} w_{X}(p)\right| d u .
\end{aligned}
$$


Combining these two estimates, we see that

$$
\sum_{p \leq X^{3}} \frac{w_{X}(p)}{p^{i t}}\left(p^{-\sigma_{X, t}}-p^{-1 / 2}\right) \ll\left(\sigma_{X, t}-\frac{1}{2}\right) X^{\sigma_{X, t}-1 / 2} \int_{1 / 2}^{\infty} X^{1 / 2-u}\left|\sum_{p \leq X^{3}} \frac{w_{X}(p) \log p \log X p}{p^{u+i t}}\right| d u .
$$

Finally, to complete the proof we bound $F(t ; X) E_{1}(t ; X)$ by (4.4) plus $F(t ; X) \log T$. To do this observe that

$$
\begin{aligned}
F(t ; X)\left|\sum_{n \leq X^{3}} \frac{\Lambda(n)}{n^{\sigma_{X, t}+i t}} w_{X}(n)\right| \leq & F(t ; X)\left|\sum_{p \leq X^{3}} \frac{\log p}{p^{\sigma_{X, t}+i t}} w_{X}(p)\right| \\
& +F(t ; X)\left|\sum_{p \leq X^{3 / 2}} \frac{\log p}{p^{2 \sigma_{X, t}+i 2 t}} w_{X}\left(p^{2}\right)\right|+O(F(t ; X)) .
\end{aligned}
$$

Trivially, the second and third terms on the right-hand side above are $\ll F(t ; X) \log T$. Finally, by (4.5) we have

$$
\left|\sum_{p \leq X^{3}} \frac{\log p}{p^{\sigma_{X, t}+i t}} w_{X}(p)\right| \ll X^{\sigma_{X, t}-1 / 2} \int_{1 / 2}^{\infty} X^{1 / 2-u}\left|\sum_{p \leq X^{3}} \frac{w_{X}(p) \log p \log X p}{p^{u+i t}}\right| d u .
$$

We now cite an inequality due to B. Hough. This enables us to establish an unconditional upper bound on the distribution function of $\log \left|\zeta\left(\frac{1}{2}+i g\right)\right| \Psi^{-1 / 2}$.

Lemma 4.3. Suppose $t>10$. For $t \neq \gamma$ we have

$$
\log \left|\zeta\left(\frac{1}{2}+i t\right)\right| \leq \log \left|\zeta\left(\sigma_{X, t}+i t\right)\right|+\frac{1}{2}\left(\sigma_{X, t}-\frac{1}{2}\right) \log t+O(1) .
$$

Proof. See Proposition 4.1 of [8]. We have applied Stirling's formula to the gamma function term.

Hough's inequality is similar to one of Soundararajan [14] (see the main proposition). Crucially, the bound here does not depend upon the truth of the Riemann hypothesis.

\section{Controlling the Error Term in the Approximate Formula}

In the error term in the approximate formula for $\log \left|\zeta\left(\frac{1}{2}+i t\right)\right|$, the term $\log ^{+}\left(1 /\left(\eta_{t} \log X\right)\right)$ will be quite large when $t$ is near an ordinate of a zero of $\zeta(s)$. Consequently, the error in approximating $\log \left|\zeta\left(\frac{1}{2}+i g\right)\right|$ may be quite large for any given $g$. However, in this section we will show that Hypothesis S implies that this can only happen for at most $o(T \log T)$ of the shifted Gram points with $T<g \leq 2 T$. We then show that for the remaining $T<g \leq 2 T$, the error term in the approximate formula for $\log \left|\zeta\left(\frac{1}{2}+i g\right)\right|$ is relatively small on average.

We first introduce some notation. We denote by $g^{*}$ any $g$ satisfying $\eta_{g} \geq 1 /(\log (|g|+2) \log \log (|g|+3))$. We denote all other $g$ by $g_{*}$.

Lemma 5.1. Assume Hypothesis S. Then as $T \rightarrow \infty$

$$
\sum_{T<g_{*} \leq 2 T} 1=o(T \log T)
$$

Proof. The proof is by contradiction. Suppose for some integer $M \geq 2$ there is a sequence $\left\{T_{n}\right\}$ such that $T_{n} \rightarrow \infty$ as $n \rightarrow \infty$, and that for each $n$

$$
\frac{1}{N_{g}\left(T_{n}, 2 T_{n}\right)} \sum_{T_{n}<g_{*} \leq 2 T_{n}} 1 \geq \frac{1}{M} .
$$

Let

$$
A_{g_{*}}=A_{g_{\ell}}=\left\{g_{\ell}, \ldots, g_{\ell+M}\right\}
$$


By Lemma 3.4 if $T_{n}<g_{*}^{\prime}<g_{*} \leq 2 T_{n}$ and $A_{g_{*}} \cap A_{g_{*}^{\prime}} \neq \emptyset$ then there is an $m \in \mathbb{N}$ with $1 \leq m \leq M$ such that

$$
\left|\left(g_{*}-g_{*}^{\prime}\right) \frac{\log T_{n}}{2 \pi}-m\right| \leq \frac{C m}{\log T_{n}}=\varepsilon_{1},
$$

where $C$ is an absolute constant. Thus, for all sufficiently large $n$,

$$
\sum_{m=1}^{M} \sum_{T_{n}<g_{*}, g_{*}^{\prime} \leq 2 T_{n}} \mathbf{1}_{\left[m-\varepsilon_{1}, m+\varepsilon_{1}\right]}\left(\left(g_{*}-g_{*}^{\prime}\right) \frac{\log T_{n}}{2 \pi}\right) \geq \sum_{\substack{T_{n}<g_{*}, g_{*}^{\prime} \leq 2 T_{n} \\ g_{*}^{\prime}<g_{*}, A_{g_{*}} \cap A_{g_{*}^{\prime}} \neq \emptyset}} 1 .
$$

To obtain a lower bound for the sum on the right-hand side, we begin by noting that by inclusion-exclusion,

$$
\left|\bigcup_{T_{n}<g_{*} \leq 2 T_{n}} A_{g_{*}}\right|-\sum_{T_{n}<g_{*} \leq 2 T_{n}}\left|A_{g_{*}}\right|+\sum_{\substack{T_{n}<g_{*}, g_{*}^{\prime} \leq 2 T_{n} \\ g_{*}^{\prime}<g_{*}}}\left|A_{g_{*}} \cap A_{g_{*}^{\prime}}\right| \geq 0 .
$$

We have $\left|A_{g_{*}}\right|=M+1,\left|A_{g_{*}} \cap A_{g_{*}^{\prime}}\right| \leq M$ for $g_{*} \neq g_{*}^{\prime}$, and $\left|\cup_{T_{n}<g_{*} \leq 2 T_{n}} A_{g_{*}}\right| \leq N_{g}\left(T_{n}, 2 T_{n}\right)+M$. Thus,

$$
M \sum_{\substack{T_{n}<g_{*}, g_{*}^{\prime} \leq 2 T_{n} \\ g_{*}^{\prime}<g_{*}, A_{g_{*}} \cap A_{g_{*}^{\prime}} \neq \emptyset}} 1 \geq \sum_{\substack{T_{n}<g_{*}^{\prime}, g_{*}^{\prime} \leq 2 T_{n} \\ g_{*}^{\prime}<g_{*}}}\left|A_{g_{*}} \cap A_{g_{*}^{\prime}}\right| \geq(M+1) \sum_{T_{n}<g_{*} \leq 2 T_{n}} 1-N_{g}\left(T_{n}, 2 T_{n}\right)-M .
$$

From this and (5.1) we obtain

$$
\sum_{\substack{T_{n}<g_{*}, g_{*}^{\prime} \leq 2 T_{n} \\ g_{*}^{\prime}<g_{*}, A_{g_{*}} \cap A_{g_{*}^{\prime}} \neq \emptyset}} 1 \geq \frac{1}{M^{2}} N_{g}\left(T_{n}, 2 T_{n}\right)-1 .
$$

Combining this with (5.2) we now see that

$$
\sum_{m=1}^{M} \sum_{T_{n}<g_{*}, g_{*}^{\prime} \leq 2 T_{n}} \mathbf{1}_{\left[m-\varepsilon_{1}, m+\varepsilon_{1}\right]}\left(\left(g_{*}-g_{*}^{\prime}\right) \frac{\log T_{n}}{2 \pi}\right) \geq \frac{1}{M^{2}} N_{g}\left(T_{n}, 2 T_{n}\right)-1 .
$$

By the definition of $g_{*}$ we know that there is an ordinate of a zero of $\zeta(s) \gamma$ so that $\left|g_{*}-\gamma\right| \leq$ $1 /\left(\log T_{n} \log \log T_{n}\right)=\varepsilon_{2}$ for $g_{*}>T_{n}$. Hence, for $T_{n}<g_{*}, g_{*}^{\prime} \leq 2 T_{n}$

$$
\left|\left(g_{*}-g_{*}^{\prime}\right) \frac{\log T_{n}}{2 \pi}-\left(\gamma-\gamma^{\prime}\right) \frac{\log T_{n}}{2 \pi}\right| \leq \frac{1}{\pi \log \log T_{n}} .
$$

Now let $\varepsilon_{3}=\varepsilon_{1}+1 /\left(\pi \log \log T_{n}\right)$. We have

$$
\sum_{T_{n}<g_{*}, g_{*}^{\prime} \leq 2 T_{n}} \mathbf{1}_{\left[m-\varepsilon_{1}, m+\varepsilon_{1}\right]}\left(\left(g_{*}-g_{*}^{\prime}\right) \frac{\log T_{n}}{2 \pi}\right) \leq \sum_{T_{n}-\varepsilon_{2}<\gamma, \gamma^{\prime} \leq 2 T_{n}+\varepsilon_{2}} \mathbf{1}_{\left[m-\varepsilon_{3}, m+\varepsilon_{3}\right]}\left(\left(\gamma-\gamma^{\prime}\right) \frac{\log T_{n}}{2 \pi}\right) .
$$

Note that

$$
\begin{aligned}
& \sum_{T_{n}-\varepsilon_{2}<\gamma, \gamma^{\prime} \leq 2 T_{n}+\varepsilon_{2}} \mathbf{1}_{\left[m-\varepsilon_{3}, m+\varepsilon_{3}\right]}\left(\left(\gamma-\gamma^{\prime}\right) \frac{\log T_{n}}{2 \pi}\right)=\sum_{T_{n}<\gamma, \gamma^{\prime} \leq 2 T_{n}} \mathbf{1}_{\left[m-\varepsilon_{3}, m+\varepsilon_{3}\right]}\left(\left(\gamma-\gamma^{\prime}\right) \frac{\log T_{n}}{2 \pi}\right) \\
& +O\left(\log ^{2} T_{n}\right),
\end{aligned}
$$

since $N(t+1)-N(t)=O(\log (|t|+2))$ (see [16] Chapter IX). Now let $\epsilon>0$. Then for all $n$ sufficiently large, we have

$$
\sum_{T_{n}<\gamma, \gamma^{\prime} \leq 2 T_{n}} \mathbf{1}_{\left[m-\varepsilon_{3}, m+\varepsilon_{3}\right]}\left(\left(\gamma-\gamma^{\prime}\right) \frac{\log T_{n}}{2 \pi}\right) \leq \sum_{T_{n}<\gamma, \gamma^{\prime} \leq 2 T_{n}} \mathbf{1}_{[m-\epsilon, m+\epsilon]}\left(\left(\gamma-\gamma^{\prime}\right) \frac{\log T_{n}}{2 \pi}\right) .
$$

By (5.4), (5.5), and (5.6) we see that

$$
\sum_{T_{n}<g_{*}, g_{*}^{\prime} \leq 2 T_{n}} \mathbf{1}_{\left[m-\varepsilon_{1}, m+\varepsilon_{1}\right]}\left(\left(g_{*}-g_{*}^{\prime}\right) \frac{\log T_{n}}{2 \pi}\right) \leq \sum_{T_{n}<\gamma, \gamma^{\prime} \leq 2 T_{n}} \mathbf{1}_{[m-\epsilon, m+\epsilon]}\left(\left(\gamma-\gamma^{\prime}\right) \frac{\log T_{n}}{2 \pi}\right)+O\left(\log ^{2} T_{n}\right) .
$$


Applying this in (5.3) we see that

$$
\sum_{m=1}^{M} \sum_{T_{n}<\gamma, \gamma^{\prime} \leq 2 T_{n}} \mathbf{1}_{[m-\epsilon, m+\epsilon]}\left(\left(\gamma-\gamma^{\prime}\right) \frac{\log T_{n}}{2 \pi}\right) \geq \frac{1}{M^{2}} N_{g}\left(T_{n}, 2 T_{n}\right)(1+o(1)) .
$$

By Hypothesis S it follows from this that

$$
\begin{aligned}
& \frac{1}{M^{2}} \leq \lim _{\epsilon \rightarrow 0^{+}}\left(\limsup _{n \rightarrow \infty} \sum_{m=1}^{M} \frac{1}{N_{g}\left(T_{n}, 2 T_{n}\right)} \sum_{T_{n}<\gamma, \gamma^{\prime} \leq 2 T_{n}} \mathbf{1}_{[m-\epsilon, m+\epsilon]}\left(\left(\gamma-\gamma^{\prime}\right) \frac{\log T_{n}}{2 \pi}\right)\right) \\
& =\sum_{m=1}^{M} \lim _{\epsilon \rightarrow 0^{+}}\left(\limsup _{n \rightarrow \infty} \frac{\left|\left\{T_{n}<\gamma, \gamma^{\prime} \leq 2 T_{n}:\left|\frac{\left(\gamma-\gamma^{\prime}\right) \log T_{n}}{2 \pi}-m\right|<\epsilon\right\}\right|}{N_{g}\left(T_{n}, 2 T_{n}\right)}\right)=0
\end{aligned}
$$

so we have reached a contradiction.

The following lemma will allow us to show that the error term in the approximate formula for $\log \mid \zeta(1 / 2+$ $\left.i g^{*}\right) \mid$ with $T<g^{*} \leq 2 T$ is relatively small on average.

Lemma 5.2. Let $X=T^{1 / 100}$ and $F(t ; X)$ be as defined in (4.1). Then

$$
\sum_{T<g^{*} \leq 2 T} F\left(g^{*} ; X\right) \ll T \log \log \log T
$$

and

$$
\sum_{T<g^{*} \leq 2 T} X^{2 \sigma_{X, g^{*}-1}} F\left(g^{*} ; X\right)^{2} \ll T \frac{(\log \log \log T)^{2}}{\log T}
$$

Proof. Recall that

$$
F(t ; X)=\left(\frac{X^{\left(1 / 2-\sigma_{X, t}\right) / 2}}{\log X}+\left(\sigma_{X, t}-\frac{1}{2}\right)\right)\left(\left(\sigma_{X, t}-\frac{1}{2}\right) \log X+\log ^{+} \frac{1}{\eta_{t} \log X}\right) .
$$

By definition $\eta_{g^{*}} \gg 1 /(\log T \log \log T)$ for $T<g^{*} \leq 2 T$ and $\sigma_{X, t} \geq 1 / 2+4 / \log X$. So that

$$
F\left(g^{*} ; X\right) \ll\left(\sigma_{X, g^{*}}-\frac{1}{2}\right)^{2} \log X+\left(\sigma_{X, g^{*}}-\frac{1}{2}\right) \log \log \log T .
$$

Both assertions of the lemma now follow from Lemma 3.6.

\section{The Proofs of Theorem 2.1 and Theorem 2.2}

Let

$$
N_{g}^{*}(T, 2 T)=\sum_{\substack{T<g \leq 2 T \\ g \neq \gamma}} 1
$$

and

$$
\mathcal{F}_{T}(v)=\frac{\left|\left\{T<g \leq 2 T, g \neq \gamma: \log \left|\zeta\left(\frac{1}{2}+i g\right)\right| \Psi^{-1 / 2} \leq v\right\}\right|}{N_{g}^{*}(T, 2 T)} .
$$

Note that $\mathcal{F}_{T}(v)$ is a distribution function. It also follows that the characteristic function corresponding to $\mathcal{F}_{T}(v)$ is given by

$$
\phi_{T}(u):=\int_{-\infty}^{\infty} e^{i u v} d \mathcal{F}_{T}(v)=\frac{1}{N_{g}^{*}(T, 2 T)} \sum_{\substack{T<g \leq 2 T \\ g \neq \gamma}} \exp \left(i u \log \left|\zeta\left(\frac{1}{2}+i g\right)\right| \Psi^{-1 / 2}\right) .
$$

Similarly, the distribution function for $\log \left|\zeta\left(\sigma_{X, g}+i g\right)\right| \Psi^{-1 / 2}$ at the points $T<g \leq 2 T$ has the corresponding characteristic function

$$
\varphi_{T}(u)=\frac{1}{N_{g}(T, 2 T)} \sum_{T<g \leq 2 T} \exp \left(i u \log \left|\zeta\left(\sigma_{X, g}+i g\right)\right| \Psi^{-1 / 2}\right) .
$$


(By the definition of $\sigma_{X, t}$ we know that $\sigma_{X, g}+i g$ is not a zero of $\zeta(s)$.)

Given distribution functions $\mathcal{F}_{1}, \mathcal{F}_{2}, \ldots$ and the corresponding characteristic functions $\phi_{1}, \phi_{2}, \ldots$ Lévy's continuity theorem states, in particular, that if $\phi_{n}$ converges pointwise on $\mathbb{R}$ to a function $\phi$ that is continuous at 0 as $n \rightarrow \infty$ then $\mathcal{F}_{n}$ converges weakly to a distribution function $\mathcal{F}$ as $n \rightarrow \infty$. Moreover, the characteristic function of $\mathcal{F}$ is $\phi$. See Billingsley [2] Theorem 26.3 or Theorem 3 from Chapter III.2 of Tenenbaum [15]. Also, note that an analogue of Lévy's continuity theorem holds when one replaces $\left\{\mathcal{F}_{n}\right\}$ by $\left\{\mathcal{F}_{T}: T>T_{0}\right\}$ and $\left\{\phi_{n}\right\}$ by $\left\{\phi_{T}: T>T_{0}\right\}$ for constant $T_{0}$.

We shall prove

Proposition 6.1. Let $X=T^{1 / 100}$. For $|u| \leq \Psi^{1 / 2} / 100$, we have

$$
\begin{aligned}
\frac{1}{N_{g}(T, 2 T)} \sum_{T<g \leq 2 T} \exp \left(i u \log \left|\zeta\left(\sigma_{X, g}+i g\right)\right| \Psi^{-1 / 2}\right) & =e^{-u^{2} / 2}\left(1+O\left(\frac{u^{2} \log \log \log T}{\Psi}\right)\right) \\
& +O\left(|u|\left(\frac{\log \log \log T}{\Psi}\right)^{1 / 2}\right)+O\left(\frac{1}{\log T}\right) .
\end{aligned}
$$

Moreover, under the assumption of Hypothesis S we shall prove

Proposition 6.2. Assume Hypothesis S. Then for $|u| \leq \Psi^{1 / 2} / 100$, we have

$$
\begin{aligned}
\frac{1}{N_{g}^{*}(T, 2 T)} \sum_{\substack{T<g \leq 2 T \\
g \neq \gamma}} \exp \left(i u \log \left|\zeta\left(\frac{1}{2}+i g\right)\right| \Psi^{-1 / 2}\right)= & e^{-u^{2} / 2}\left(1+O\left(\frac{u^{2} \log \log \log T}{\Psi}\right)\right) \\
& +o(1)+O\left(|u| \frac{\log \log \log T}{\Psi^{1 / 2}}\right) .
\end{aligned}
$$

Note that $e^{-u^{2} / 2}$ is the characteristic function of a normally distributed random variable with mean zero and variance one. Hence, Theorem 2.2 immediately follows from Proposition 6.2 and and Lévy's continuity theorem.

We will now deduce Theorem 2.1 using Proposition 6.1 and Lemma 4.3.

Proof of Theorem 2.1. By Lemma 4.3 there is an absolute constant $C>0$ such that for $T<t \leq 2 T$ we have for $t \neq \gamma$ that

$$
\log \left|\zeta\left(\frac{1}{2}+i t\right)\right| \leq \log \left|\zeta\left(\sigma_{X, t}+i t\right)\right|+\frac{1}{2}\left(\sigma_{X, t}-\frac{1}{2}\right) \log T+C .
$$

Writing $\Delta(t)=\left(\left(\sigma_{X, t}-\frac{1}{2}\right) \log T+C\right) \Psi^{-1 / 2}$ we see that

$$
\sum_{\substack{T<g \leq 2 T \\ g \neq \gamma}} \mathbf{1}_{[\alpha, \infty)}\left(\log \left|\zeta\left(\frac{1}{2}+i g\right)\right| \Psi^{-1 / 2}\right) \leq \sum_{T<g \leq 2 T} \mathbf{1}_{[\alpha-\Delta(g), \infty)}\left(\log \left|\zeta\left(\sigma_{X, g}+i g\right)\right| \Psi^{-1 / 2}\right) .
$$

Using Lemma 3.6 and Chebyshev's inequality we have for any fixed $\epsilon>0$

$$
\sum_{\substack{T<g \leq 2 T \\ \Delta(g) \geq \epsilon}} 1 \leq \epsilon^{-1} \sum_{T<g \leq 2 T} \Delta(g) \ll \frac{T}{\epsilon \Psi^{1 / 2}} \log T=o(T \log T) .
$$

Thus,

$$
\sum_{T<g \leq 2 T} \mathbf{1}_{[\alpha-\Delta(g), \infty)}\left(\log \left|\zeta\left(\sigma_{X, g}+i g\right)\right| \Psi^{-1 / 2}\right) \leq \sum_{T<g \leq 2 T} \mathbf{1}_{[\alpha-\epsilon, \infty)}\left(\log \left|\zeta\left(\sigma_{X, g}+i g\right)\right| \Psi^{-1 / 2}\right)+o(T \log T) .
$$

Hence, Proposition 6.1 and Lévy's continuity theorem imply that

$$
\frac{1}{N_{g}(T, 2 T)} \sum_{T<g \leq 2 T} \mathbf{1}_{[\alpha-\epsilon, \infty)}\left(\log \left|\zeta\left(\sigma_{X, g}+i g\right)\right| \Psi^{-1 / 2}\right)=\frac{1}{\sqrt{2 \pi}} \int_{a-\epsilon}^{\infty} e^{-x^{2} / 2} d x+o(1) .
$$

Since $\epsilon$ is arbitrary Theorem 2.1 now follows from this, (6.1) and (6.2). 
Lemma 6.3. Let $X=T^{1 / 100}$. For $|u| \leq \Psi^{1 / 2} / 100$, we have

$$
\begin{aligned}
\frac{1}{N_{g}(T, 2 T)} \sum_{T<g \leq 2 T} \exp \left(i u \sum_{p \leq X^{3}} \frac{\cos (g \log p)}{p^{1 / 2}} \Psi^{-1 / 2}\right)= & e^{-u^{2} / 2}\left(1+O\left(\frac{u^{2} \log \log \log T}{\Psi}\right)\right) \\
& +O\left(|u|\left(\frac{\log \log \log T}{\Psi}\right)^{1 / 2}\right)+O(1 / \log T) .
\end{aligned}
$$

Proof. By Stieltjes integration

$$
\sum_{T<g \leq 2 T} \exp \left(i u \sum_{p \leq X^{3}} \frac{\cos (g \log p)}{p^{1 / 2}} \Psi^{-1 / 2}\right)=\int_{T}^{2 T} \exp \left(i u \sum_{p \leq X^{3}} \frac{\cos (t \log p)}{p^{1 / 2}} \Psi^{-1 / 2}\right) d \mu(t),
$$

where $\mu(t)=\mu_{\phi}(t)=\lfloor(\theta(t)+\phi) / \pi\rfloor=t /(2 \pi) \log (t /(2 \pi e))+r(t)$ and $r(t) \ll 1$ for $t \geq 10$. The right-hand side of this equals

$$
\int_{T}^{2 T} \exp \left(i u \sum_{p \leq X^{3}} \frac{\cos (t \log p)}{p^{1 / 2}} \Psi^{-1 / 2}\right) d\left(\frac{t}{2 \pi} \log \frac{t}{2 \pi e}\right)+\int_{T}^{2 T} \exp \left(i u \sum_{p \leq X^{3}} \frac{\cos (t \log p)}{p^{1 / 2}} \Psi^{-1 / 2}\right) d r(t) .
$$

The first integral equals

$$
\frac{1}{2 \pi} \int_{T}^{2 T} \exp \left(i u \sum_{p \leq X^{3}} \frac{\cos (t \log p)}{p^{1 / 2}} \Psi^{-1 / 2}\right) \log \frac{t}{2 \pi} d t
$$

which by the Second Mean Value Theorem equals

$$
\frac{1}{2 \pi} \log \frac{T}{2 \pi} \int_{T}^{2 T} \exp \left(i u \sum_{p \leq X^{3}} \frac{\cos (t \log p)}{p^{1 / 2}} \Psi^{-1 / 2}\right) d t+O(T) .
$$

By Lemma 3.9 this equals

$$
\frac{T}{2 \pi} \log \frac{T}{2 \pi} e^{-u^{2} / 2}\left(1+O\left(\frac{u^{2} \log \log \log T}{\Psi}\right)\right)+O\left(|u| T \log T\left(\frac{\log \log \log T}{\Psi}\right)^{1 / 2}\right)+O(T)
$$

for $|u| \leq \Psi^{1 / 2} / 100$.

It remains to bound the second integral in (6.3). Integrating by parts we see that

$$
\int_{T}^{2 T} \exp \left(i u \sum_{p \leq X^{3}} \frac{\cos (t \log p)}{p^{1 / 2}} \Psi^{-1 / 2}\right) d r(t) \ll 1+\left|\int_{T}^{2 T} r(t) d\left(\exp \left(i u \sum_{p \leq X^{3}} \frac{\cos (t \log p)}{p^{1 / 2}} \Psi^{-1 / 2}\right)\right)\right| .
$$

Now

$$
\int_{T}^{2 T} r(t) d\left(\exp \left(i u \sum_{p \leq X^{3}} \frac{\cos (t \log p)}{p^{1 / 2}} \Psi^{-1 / 2}\right)\right) \ll \frac{|u|}{\Psi^{1 / 2}} \int_{T}^{2 T}\left|\sum_{p \leq X^{3}} \frac{\sin (t \log p) \log p}{p^{1 / 2}}\right| d t .
$$

Applying Cauchy's inequality and then Montgomery and Vaughan's mean value theorem for Dirichlet polynomials [11], we see that

$$
\begin{aligned}
\int_{T}^{2 T}\left|\sum_{p \leq X^{3}} \frac{\sin (t \log p) \log p}{p^{1 / 2}}\right| d t & \leq T^{1 / 2}\left(\int_{T}^{2 T}\left|\sum_{p \leq X^{3}} \frac{\log p}{p^{1 / 2+i t}}\right|^{2} d t\right)^{1 / 2} \\
& \ll T^{1 / 2}\left(T \log ^{2} T\right)^{1 / 2}=T \log T
\end{aligned}
$$

We now have that

$$
\int_{T}^{2 T} \exp \left(i u \sum_{p \leq X^{3}} \frac{\cos (t \log p)}{p^{1 / 2}} \Psi^{-1 / 2}\right) d r(t) \ll 1+\frac{|u|}{\Psi^{1 / 2}} T \log T
$$


Combining this and (6.4) in (6.3) we obtain

$$
\begin{aligned}
\frac{1}{N_{g}(T, 2 T)} \sum_{T<g \leq 2 T} \exp \left(i u \sum_{p \leq X^{3}} \frac{\cos (g \log p)}{p^{1 / 2}} \Psi^{-1 / 2}\right)= & e^{-u^{2} / 2}\left(1+O\left(\frac{u^{2} \log \log \log T}{\Psi}\right)\right) \\
& +O\left(|u|\left(\frac{\log \log \log T}{\Psi}\right)^{1 / 2}\right)+O(1 / \log T)
\end{aligned}
$$

Observe that Proposition 6.1 follows immediately from Lemma 6.3 and

Lemma 6.4. Let $X=T^{1 / 100}$. For $|u| \leq \Psi^{1 / 2} / 100$, we have

$$
\begin{aligned}
\sum_{T<g^{*} \leq 2 T} \exp \left(i u \log \left|\zeta\left(\frac{1}{2}+i g^{*}\right)\right| \Psi^{-1 / 2}\right)= & \sum_{T<g^{*} \leq 2 T} \exp \left(i u \sum_{p \leq X^{3}} \frac{\cos \left(g^{*} \log p\right)}{p^{1 / 2}} \Psi^{-1 / 2}\right) \\
& +O\left(\frac{|u| T \log T \log \log \log T}{\Psi^{1 / 2}}\right) .
\end{aligned}
$$

Additionally, under the same hypotheses we have

$$
\sum_{T<g \leq 2 T} \exp \left(i u \log \left|\zeta\left(\sigma_{X, g}+i g\right)\right| \Psi^{-1 / 2}\right)=\sum_{T<g \leq 2 T} \exp \left(i u \sum_{p \leq X^{3}} \frac{\cos (g \log p)}{p^{1 / 2}} \Psi^{-1 / 2}\right)+O\left(\frac{|u| T \log T}{\Psi^{1 / 2}}\right) .
$$

Proof. We will omit the proof of the second assertion as it follows from a similar argument. Note that in the second statement the error term is slightly smaller because we can obtain better estimates on averages of $X^{\left(\sigma_{X, g}-\frac{1}{2}\right)}$ and $\left(\sigma_{X, g}-\frac{1}{2}\right)$ as opposed to averages of $F\left(g^{*} ; X\right)$. (Compare Lemma 3.6 to Lemma 5.2.)

Note that $\left|e^{i a}-e^{i b}\right|=\left|\int_{a}^{b} e^{i t} d t\right| \leq|b-a|$. By this and Lemma 4.2 we have

$$
\begin{gathered}
\sum_{T<g^{*} \leq 2 T}\left|\exp \left(i u \log \left|\zeta\left(\frac{1}{2}+i g^{*}\right)\right| \Psi^{-1 / 2}\right)-\exp \left(i u \sum_{p \leq X^{3}} \frac{\cos \left(g^{*} \log p\right)}{p^{1 / 2}} \Psi^{-1 / 2}\right)\right| \\
\ll \frac{|u|}{\Psi^{1 / 2}} \sum_{T<g^{*} \leq 2 T}\left(F\left(g^{*} ; X\right) X^{\sigma_{X, g^{*}-1 / 2}} \int_{1 / 2}^{\infty} X^{1 / 2-u}\left|\sum_{p \leq X^{3}} \frac{\log p \log p X}{p^{u+i g^{*}}} w_{X}(p)\right| d u\right. \\
\left.+F\left(g^{*} ; X\right) \log T+E_{2}\left(g^{*} ; X\right)+E_{3}\left(g^{*} ; X\right)\right) .
\end{gathered}
$$

(See (4.2) for the definition of $E_{1}$ and Lemma 4.2 for the definitions of $E_{2}, E_{3}$.) Observe that $\left(1-w_{X}(p)\right) \ll$ $\log p / \log X$, so by Cauchy's inequality and (3.3),

$$
\sum_{T<g^{*} \leq 2 T} E_{2}\left(g^{*} ; X\right) \ll(T \log T)^{1 / 2}\left(\sum_{T<g \leq 2 T} E_{2}(g ; X)^{2}\right)^{1 / 2} \ll T \log T .
$$

Similarly, by (3.4)

$$
\sum_{T<g^{*} \leq 2 T} E_{3}\left(g^{*} ; X\right) \ll(T \log T)^{1 / 2}\left(\sum_{T<g \leq 2 T} E_{3}(g ; X)^{2}\right)^{1 / 2} \ll T \log T .
$$

Next, by Lemma 5.2

$$
\sum_{T<g^{*} \leq 2 T} F\left(g^{*} ; X\right) \log T \ll T \log T(\log \log \log T) .
$$

It remains to bound the first term in (6.6). We begin by applying Cauchy's inequality to see that

$$
\begin{aligned}
& \sum_{T<g^{*} \leq 2 T} F\left(g^{*} ; X\right) X^{\sigma_{X, g^{*}-1 / 2}} \int_{1 / 2}^{\infty} X^{1 / 2-u}\left|\sum_{p \leq X^{3}} \frac{\log p \log p X}{p^{u+i g^{*}}} w_{X}(p)\right| d u \\
\leq & \left(\sum_{T<g^{*} \leq 2 T} F^{2}\left(g^{*} ; X\right) X^{2 \sigma_{X, g^{*}-1}}\right)^{1 / 2}\left(\sum_{T<g^{*} \leq 2 T}\left(\int_{1 / 2}^{\infty} X^{1 / 2-u}\left|\sum_{p \leq X^{3}} \frac{\log p \log p X}{p^{u+i g^{*}}} w_{X}(p)\right| d u\right)^{2}\right)^{1 / 2} .
\end{aligned}
$$


By Lemma 5.2,

$$
\sum_{T<g^{*} \leq 2 T} F^{2}\left(g^{*} ; X\right) X^{2 \sigma_{X, g^{*}-1}} \ll T \frac{(\log \log \log T)^{2}}{\log T} .
$$

Next, apply Cauchy's inequality to the final factor on the right-hand side of (6.7) to see that

$$
\begin{aligned}
\sum_{T<g^{*} \leq 2 T}\left(\int_{1 / 2}^{\infty} X^{1 / 2-u}\right. & \left.\left|\sum_{p \leq X^{3}} \frac{\log p \log p X}{p^{u+i g^{*}}} w_{X}(p)\right| d u\right)^{2} \\
\leq & \left(\int_{1 / 2}^{\infty} X^{1 / 2-u} d u\right)\left(\int_{1 / 2}^{\infty} X^{1 / 2-u} \sum_{T<g^{*} \leq 2 T}\left|\sum_{p \leq X^{3}} \frac{\log p \log p X}{p^{u+i g^{*}}} w_{X}(p)\right|^{2} d u\right) \\
& \ll \int_{1 / 2}^{\infty} X^{1 / 2-u} \log ^{3} X \sum_{T<g \leq 2 T}\left|\sum_{p \leq X^{3}} \frac{\log p \log p X}{p^{u+i g} \log ^{2} X} w_{X}(p)\right|^{2} d u .
\end{aligned}
$$

Noting that for $p \leq X^{3}$ we have $\log p \log p X /\left(p^{u} \log ^{2} X\right) \ll \log p /\left(p^{1 / 2} \log X\right)$ for $u \geq 1 / 2$, we see from (3.3) that the sum over $g$ is $\ll T \log T$ uniformly for $u \geq 1 / 2$. Hence, the quantity above is $\ll T \log ^{3} T$. Combining this with (6.7) and (6.8) we see that

$$
\sum_{T<g^{*} \leq 2 T} F\left(g^{*} ; X\right) X^{\sigma_{X, g^{*}-1 / 2}} \int_{1 / 2}^{\infty} X^{1 / 2-u}\left|\sum_{p \leq X^{3}} \frac{\log p \log p X}{p^{u+i g^{*}}} w_{X}(p)\right| d u \ll T \log T \log \log \log T .
$$

Proof of Proposition 6.2. Since $\left|e^{i t}\right|=1$ we see that by Lemma 5.1 extending the range of the sum on the lefthand side of (6.5) to $T<g \leq 2 T, g \neq \gamma$ produces an error term of size at most $o(T \log T)$. Similarly, extending the sum on the right-hand side of (6.5) to all of $T<g \leq 2 T$ gives an error term not exceeding $o(T \log T)$. Also, by Lemma $5.1 N_{g}^{*}(T, 2 T)=N_{g}(T, 2 T)(1+o(1))$. Hence, by these observations we have for $|u| \leq \Psi^{1 / 2} / 100$ that

$$
\begin{aligned}
\frac{1}{N_{g}^{*}(T, 2 T)} \sum_{\substack{T<g \leq 2 T \\
g \neq \gamma}} \exp \left(i u \log \left|\zeta\left(\frac{1}{2}+i g\right)\right| \Psi^{-1 / 2}\right)= & \frac{1}{N_{g}(T, 2 T)} \sum_{T<g \leq 2 T} \exp \left(i u \sum_{p \leq X^{3}} \frac{\cos (g \log p)}{p^{1 / 2}} \Psi^{-1 / 2}\right) \\
& +O\left(\frac{|u| \log \log \log T}{\Psi^{1 / 2}}\right)+o(1) .
\end{aligned}
$$

Applying Lemma 6.3 completes the proof.

\subsection{Acknowledgments}

The majority of this article is part of the author's PhD thesis, which was supervised by Prof. Steven Gonek. I would like to thank Prof. Gonek for suggesting this problem and also for his guidance and encouragement. The exposition of this article has greatly benefited from his advice and suggestions. I am also very grateful to the anonymous referee for giving helpful suggestions and remarks, which significantly improved this article. In particular, for pointing out the unconditional inequality of Bob Hough and suggesting Theorem 2.1. This theorem is new to this version. This work was supported in part by NSF grant DMS-1200582.

\section{References}

[1] Banks, W., V. Castillo-Garate, L. Fontana, and C. Morpurgo, "Self-intersections of the Riemann zetafunction on the critical line." Journal of Mathematical Analysis and Applications 406, no. 2 (2013): 475-481.

[2] Billingsley, P. Probability and Measure, 2nd ed. New York: John Wiley, 1986.

[3] Bombieri, E. and D. A. Hejhal. "On the zeros of Epstein zeta functions." Comptes Rendus de l'Académie des Sciences Paris Série I Mathématiques 304, no. 9 (1987): 213-217.

[4] Bombieri, E. and D. A. Hejhal. "On the distribution of zeros of linear combinations of Euler products". Duke Mathematical Journal 80, no. 3 (1995): 821-862. 
[5] Borchsenius, V. and B. Jessen. "Mean Motions and Values of the Riemann Zeta Function." Acta Mathematica 80, no. 1 (1948): 97-166.

[6] Farmer, D. W., S. M. Gonek, and Y. Lee. "Pair Correlation of the zeros of the derivative of the Riemann $\xi$-function." (2013): preprint.

[7] Hejhal, D. A. "On the distribution of $\log \left|\zeta^{\prime}\left(\frac{1}{2}+i t\right)\right| . "$ Number theory, trace formulas and discrete groups (Oslo, 1987), 343-370. Boston: Academic Press, 1989.

[8] Hough, B. "The Distribution of the logarithm of orthogonal and symplectic L-functions." (2011): preprint arXiv:1109.1783.

[9] Levinson, N. "Almost all roots of $\zeta(s)=a$ are arbitrarily close to $\sigma=1 / 2$." Proceeding of the Natural Academy of Sciences of the United States of America 72, (1975):1322-1324.

[10] Montgomery, H. L. "The pair correlation of zeros of the zeta function." In Analytic Number Theory, 181193. Proceedings of Symposia in Pure Mathematics 24. Providence, RI: American Mathematical Society, 1973.

[11] Montgomery, H. L. and R. C. Vaughan. "Hilbert's inequality." Journal of the London Mathematical Society (2) $8,(1974): 73-82$.

[12] Selberg, A. "Contributions to the theory of the Riemann zeta-function." Archiv for Mathematik og Naturvidenskab 48, no. 5 (1946): 89-155.

[13] Selberg, A. "Old and new conjectures and results about a class of Dirichlet series." In Proceedings of the Amalfi Conference on Analytic Number Theory (Maiori 1989),367-385. Salerno: Università di Salerno 1992.

[14] Soundararajan, K., "Moments of the Riemann zeta function", Ann. of Math. (2) 170 (2009), no. 2, 981-993.

[15] Tenenbaum, G. Introduction to analytic and probabilistic number theory, Cambridge: Cambridge University Press, 1995.

[16] Titchmarsh, E. C. The theory of the Riemann zeta-function, 2nd ed. Revised by D.R. Heath-Brown. Oxford: Oxford University Press, 1986.

[17] Tsang, K. M. "The distribution of the values of the Riemann zeta-function." PhD diss., Princeton Univ., Princeton, 1984. 